\title{
Counterintuitive consequence of heating in strongly-driven intrinsic junctions of $\mathrm{Bi}_{2} \mathrm{Sr}_{2} \mathrm{CaCu}_{2} \mathrm{O}_{8+\delta}$ mesas
}

\author{
C. Kurter,,${ }^{1,2, *}$ L. Ozyuzer, ${ }^{1,3}$ T. Proslier, ${ }^{1,2}$ J. F. Zasadzinski, ${ }^{2}$ D. G. Hinks, ${ }^{1}$ and K. E. Gray ${ }^{1}$ \\ ${ }^{1}$ Materials Science Division, Argonne National Laboratory, Argonne, Illinois 60439, USA \\ ${ }^{2}$ Physics Division, BCPS Department, Illinois Institute of Technology, Chicago, Illinois 60616, USA \\ ${ }^{3}$ Department of Physics, Izmir Institute of Technology, TR-35430 Izmir, Turkey \\ (Received 9 November 2009; revised manuscript received 7 June 2010; published 22 June 2010)
}

\begin{abstract}
Anomalously high and sharp peaks in the conductance of intrinsic Josephson junctions in $\mathrm{Bi}_{2} \mathrm{Sr}_{2} \mathrm{CaCu}_{2} \mathrm{O}_{8+\delta}$ (Bi2212) mesas have been commonly interpreted as superconducting energy gaps but here we show they are a result of strong self-heating. This conclusion follows directly from a comparison to the equilibrium gap measured by tunneling in single break junctions on equivalent crystals. As the number of junctions in the mesa, $N$, and thus heating increase, the peak voltages decrease and the peak width abruptly sharpens for $N \geq 12$. Clearly these widely variable features vs $N$ cannot all represent the equilibrium properties. Our data imply that the sharp peaks represent a transition to the normal state. That it occurs at the same dissipated power for $N$ $=12-30$ strongly implicates heating as the cause. Although peak sharpening due to heating is counterintuitive, as tunneling spectra usually broaden at higher temperatures, a lateral temperature gradient, leading to coexistence of normal hot spots and superconductive regions, qualitatively explains the behavior. However, a more uniform temperature profile cannot be ruled out. As the peak's width and voltage in our shortest mesa $(N$ =6) are more consistent with the break junction data, we propose a figure of merit for Bi2212 mesas, the relative conductance peak width, such that small values signal a crossover into the strong self-heating regime.
\end{abstract}

DOI: $10.1103 /$ PhysRevB.81.224518

PACS number(s): 74.50. $+\mathrm{r}$

\section{INTRODUCTION}

Intrinsic Josephson junctions (IJJs) in the crystal structure of the high-temperature superconductor $\mathrm{Bi}_{2} \mathrm{Sr}_{2} \mathrm{CaCu}_{2} \mathrm{O}_{8+\delta}$ (Bi2212) exist along the $c$ axis between each pair of neighboring $\mathrm{CuO}_{2}$ bilayers, with the $\mathrm{Bi}_{2} \mathrm{Sr}_{2} \mathrm{O}_{4}$ layers acting as the insulating tunnel barrier. Kleiner et al., ${ }^{1}$ described the novel features of such stacks of superconductor-insulatorsuperconductor (SIS) Josephson junctions and continuing excitement about research prospects has led to an exhaustive literature on the current-voltage characteristics, $I(\mathrm{~V})$, of IJJs, especially on sculpted mesas. ${ }^{2-16} \mathrm{~A}$ stack of IJJs, in a mesa sculpted on a Bi2212 single crystal, offers a unique possibility for spectroscopy and exotic device arrays using welldefined, uniform tunnel barriers that are stable over the entire temperature range. However, the poor thermal conductivity, very large current density, and close proximity of multiple neighboring junctions in the crystal structure of Bi2212 have led to significant concerns about heating. A striking example is found in large volume mesas as a backbending of the $I(V)$, e.g., Figs. 1(a) and 1(b), occurring at voltages well below the gap voltage, $2 \Delta / e$. The cause of such backbending was shown ${ }^{4,16-18}$ to be self-heating that is dominated by the particular temperature dependence of the subgap quasiparticle $c$-axis resistivity, $\rho_{\mathrm{c}}\left(T_{\mathrm{m}}\right)$, where $T_{\mathrm{m}}$ is the temperature of the heated mesa. Despite such heating concerns, numerous fundamental studies ${ }^{2-14}$ on Bi2212 mesas have drawn conclusions about the magnitude and temperature dependence of the superconducting gap, $\Delta(T)$, in Bi2212 along with inferences about an extrinsic pseudogap.

Interest in Bi2212 IJJ stacks has been further peaked by the recent demonstration of emission of significant terahertzwave radiation. ${ }^{18}$ In order to achieve the needed terahertz cavity resonance and high emission power, large mesa vol- umes were used and those are at odds with conventional trend in Bi2212 mesa research to reduce heating, being up to $10^{4}-10^{6}$ times larger in volume. Thus the ongoing quest to

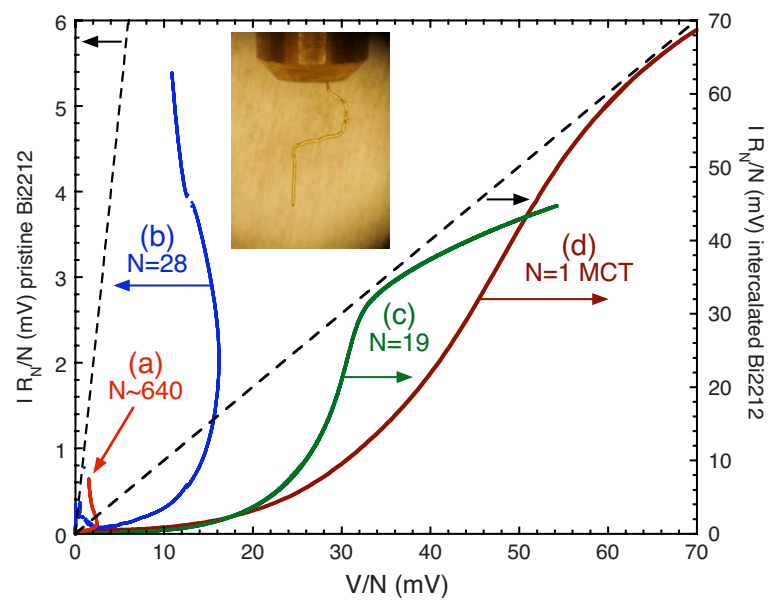

FIG. 1. (Color online) Comparison of tunneling data for three mesas and a single MCT SIS junction (d) at $4.2 \mathrm{~K}$, except (a) which is at $26 \mathrm{~K}$. Note (a) and (b) use pristine Bi2212 and are plotted on the left-hand axis while (c) and (d) use intercalated Bi2212 and are plotted on the right-hand axis. The dashed lines estimate the normal-state resistance, $R_{\mathrm{N}}$, in each case: (a) $12.8 \Omega$; (b) $30 \Omega$; (c) $460 \Omega$; and (d) $15 \mathrm{k} \Omega$. Departures from this line at higher voltages are caused by the energy dependence of the normal-state density of states and are also seen in the MCT SIN data displayed in Fig. 4. This departure is especially noticeable in (c) where the effect of heating also plays a role (see Fig. 7). Curve (a) is a 300 $\times 60 \mu \mathrm{m}^{2}$ terahertz emitting mesa of thickness $1 \mu \mathrm{m}(N \sim 640)$. Inset: the 100- $\mu \mathrm{m}$-soft $\mathrm{Au}$ wire used to contact the $\mathrm{Au}$ film atop the $10 \times 10 \mu \mathrm{m}^{2}$ mesas in (b), (c), and in Fig. 2. The hook shape minimizes contact force (damage) from the sharpened tip. 


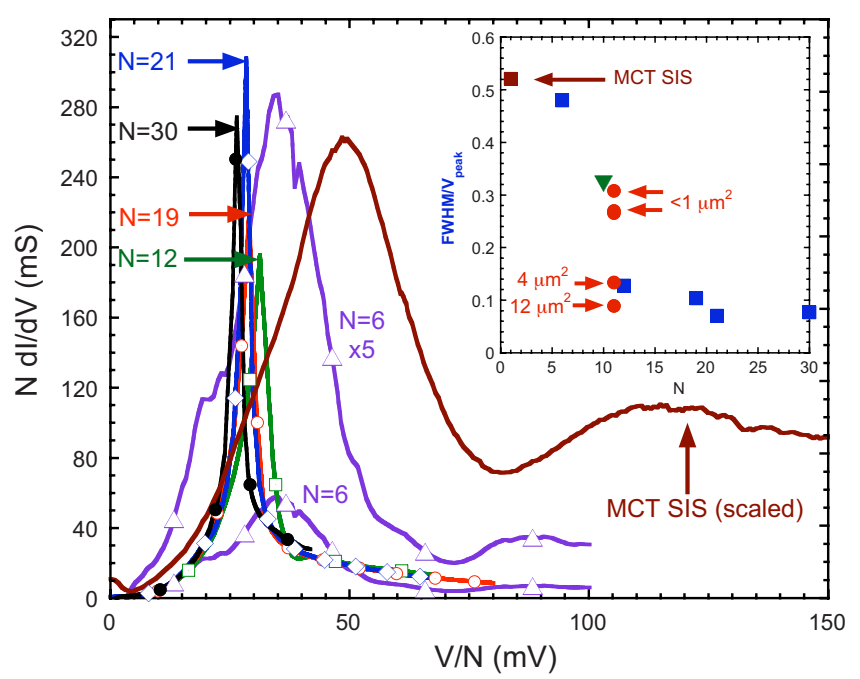

FIG. 2. (Color online) Conductance per junction, $N d I / d V$, for each intercalated mesa compared to scaled data for an intercalated MCT junction $(N=1)$. Symbols are used to identify the curves and all data taken at $T_{\mathrm{B}}=4.2 \mathrm{~K}$. Inset: ratio, $\beta$, of FWHM to the peak voltage for each, together with those calculated for Ref. 3-inverted triangle—and Ref. 7-filled circles.

understand self-heating effects in Bi2212 mesas is clearly front and center.

The recently common reports ${ }^{8-12}$ of high, sharp conductance peaks without backbending in IJJ stacks of Bi2212 mesas have led to two nonheating interpretations: (1) evidence for minimal thermal broadening of an intrinsically narrow peak in the superconducting density of states (DOS), $N(E)$, in Bi2212, where $E$ is the quasiparticle energy; and (2) evidence for a strictly coherent $c$-axis tunneling process in mesas, due to their necessarily aligned $a$ and $b$ mesa axes, which may better preserve the narrow features of $N(E)$ in the SIS conductance (see Ref. 8). Data presented here on mesas of variable heights $(13-65 \mathrm{~nm})$ together with mechanical contact tunneling (MCT) on equivalent crystals will be shown to conflict with each interpretation. For (2), the shortest mesa stack data ( $N=6 \mathrm{IJJs})$ do not exhibit sharp peaks but they are more representative of the much broader peaks seen in MCT (see Fig. 2) for which the $a$ and $b$ mesa axes alignment is unknown. Since there is no reason for the coherently aligned $a$ and $b$ axes to be lost when $N=6$, these data are inconsistent with conclusion (2). Specifically pointing out this conflict is an important contribution of the present work. In the case of interpretation (1), the MCT data shows that the equilibrium tunneling DOS peak in Bi2212 is intrinsically broader than the recent observations ${ }^{8-12}$ of sharp peaks in mesas would suggest. This eliminates the interpretation of minimal broadening of a sharp DOS in Bi2212 and suggests another effect is taking place. We will demonstrate here that similarly high, sharp conductance peaks observed in our mesas are due to strong self-heating and represent the transition of the mesa into the normal state.

The ability to compare mesa conductance spectra directly with single-junction MCT on the same (or similar) crystals is a unique aspect of this work. Note that the MCT technique has pioneered a number of spectroscopic discoveries, ${ }^{19-25}$ e.g., the dip-hump features (DHFs) in Bi2212 that are visible at 80 and $120 \mathrm{mV}$ in the MCT data of Fig. 2. These have been found subsequently, and greatly expanded upon, by angle-resolved photoemission spectroscopy (ARPES) (see, e.g., Ref. 26)] and scanning tunneling spectroscopy (STS). ${ }^{27-31}$ There is excellent agreement among all three techniques with no significant discrepancies. ${ }^{23,32}$ An important advantage of MCT and STS is a significant reduction in heating as these junctions usually exhibit much lower dissipation due to: higher specific resistance; smaller areas; and improved heat removal as they consist of only one dissipating junction, rather than a mesa stack. If one compares published data on Bi2212 mesas to these traditional singlejunction methods, the only mesas that show similar behavior, like the spectral dip-hump feature, ${ }^{23,28}$ have greatly minimized heating effects. These latter mesas use short stacks $(N \sim 10)$ with either: intercalated Bi2212 (Refs. 3 and 33) to reduce the dissipative $c$-axis quasiparticle conductance; ultrasmall areas $\left(<1 \mu \mathrm{m}^{2}\right)$ (Ref. 7) to improve heat removal; or 60 ns current pulses ${ }^{4,13-15}$ to minimize the buildup of mesa temperature, $T_{\mathrm{m}}$.

Previous authors ${ }^{4,17}$ have also questioned the validity of interpreting such sharp peaks as the superconductive energy gap. These authors have argued that sharp peaks, or the conventionally held sign of heating, backbending in $I(V)$, result from uniform heating and the temperature dependence of the quasiparticle tunneling resistance in the limit of low bias voltage, $\rho_{\mathrm{c}}(T)$. We call this type-I backbending and it arises predominantly from thermal activation of quasiparticles even without a substantial reduction in $\Delta$. However our data emulate recent studies ${ }^{8-12}$ that find (a) the absence of backbending; and (b) sharp peaks at voltages closer to $2 \Delta / e$ than the positions of previously reported cases of type-I backbending (Refs. 4 and 16-18). When heating becomes significant for $V$ closer to $2 \Delta / e$, the temperature dependence of the peak in the superconducting DOS, $N(E)$, is more relevant. We define this as type-II backbending, i.e., a case that is predominantly due to the reduction in $\Delta$ (by heating), and has been observed in low- $T_{\mathrm{c}}$ superconductors. ${ }^{34}$ Of course, intermediate cases can occur so any model developed to describe heating must include the intrinsic $N(E)$ and its temperature dependence. Unfortunately our MCT technique cannot measure the $N(E)$ at higher $T$ due to mechanical instabilities. Thus our heating analysis is restricted to the use of the $d$-wave DOS, ${ }^{35}$

$$
\left.N_{s}(E, k)=\operatorname{Re}\left\{(E-i \Gamma) / \sqrt{ }\left[(E-i \Gamma)^{2}-\Delta(k)^{2}\right]\right\}\right\},
$$

with the only available $\Delta(T)$ and $\Gamma(T)$ coming from STM studies on pristine, i.e., unintercalated, Bi2212. ${ }^{31}$ Here $E$ is the quasiparticle energy, $\Delta(k)=\Delta_{0} \cos (2 \phi)$, $\phi$ is polar angle in $k$ space, and $\Gamma$ is smearing parameter, assumed to arise from the quasiparticle scattering rate. Then $N(E)$ is the sum of $N_{s}(E, k)$ over $k$ states and its temperature dependence originates from the temperature dependencies of $\Delta$ and $\Gamma$. Proceeding in this way, we have been unable to completely eliminate backbending for all mesa heights using a reasonable value for the heat-transfer coefficient. However our model with a lateral temperature gradient across the mesa (with normal and superconducting regions) provides qualitative agreement with our data and we will argue it is likely 
closer to the actual situation. It seems that the absence of backbending is likely most strongly affected by $N(E)$ at intermediate $E$. Our low-temperature data on pristine and intercalated $\mathrm{Bi} 2212$ will be shown below to exhibit a flatter $N(E)$ than the $d$-wave DOS [Eq. (1)] and thus may be less prone to instability leading to backbending than our model. This realization means that the possibility of a fairly uniform temperature solution cannot be completely ruled out.

While a significant portion of this paper discusses detailed heating models to reproduce the measured $I(V)$ observed for different height mesas, and additional results are obtained, it must be remembered that a principal conclusion of this work can be drawn directly from the experimental data. The reasoning is as follows. Our shortest mesa $(N=6)$ exhibits the least heating and most closely resembles the SIS junction from MCT, with a relatively broader conductance peak as well as dip/hump features. As $N$ increases, Fig. 2 shows that the voltage of the conductance peak decreases and the peak abruptly sharpens for $N \geq 12$. Clearly the widely variable data for various $N$ cannot all represent equilibrium properties. The decrease in peak voltage with increasing $N$ certainly implicates strong self-heating. In addition, for $N=12-30$, the sharp peaks occur for roughly the same power dissipation $(0.5-0.6 \mathrm{~mW})$ and the return to the (normal-state) conductance (see Fig. 2) is also at the same power (1-1.1 mW) indicating these effects occur at the same $T_{\mathrm{m}}$, and that strongly implicates heating. Note that data showing similar changes in peak widths were reported as a function of mesa area, with $N=10-11 .^{7}$ In that study, mesas with the most efficient heat removal (i.e., smallest area) also recovered the broader DOS peak commonly observed with MCT. Further, the absence of any dip-hump features beyond the peak voltage for our $N=12-30$ mesas, and the larger area mesas of Ref. 7 implies that $T_{\mathrm{m}}$ is then at or near $T_{\mathrm{C}} \cdot{ }^{30}$ This data implies an effective thermal resistance, $\alpha$, of $\sim\left(T_{\mathrm{c}}\right.$ $\left.-T_{\mathrm{B}}\right) / 1 \mathrm{~mW} \sim 70 \mathrm{~K} / \mathrm{mW}$ for our $10 \times 10 \mu \mathrm{m}^{2}$ mesas.

Thus the primary result of this paper is our experimental demonstration (Fig. 2) that sharp conductance peaks similar to those in Refs. 8-12 are not a measure of a superconducting gap, $\Delta$. Rather, they represent the transition of the mesa into the normal state due to self-heating. The importance of our experimental finding relates to conclusions drawn by others about the magnitude and temperature dependence of $\Delta$, along with inferences about an extrinsic pseudogap in Bi2212, that are based on IJJ data exhibiting such sharp peaks. $^{8-12}$ Note that since high temperatures normally broaden tunneling spectra, heating is a counterintuitive conclusion to explain sharper peaks.

\section{EXPERIMENT}

Single crystals of Ca-rich $\mathrm{Bi}_{2.1} \mathrm{Sr}_{1.4} \mathrm{Ca}_{1.5} \mathrm{Cu}_{2} \mathrm{O}_{8+\delta}$, were grown by a floating-zone technique. Intercalation of $\mathrm{HgBr}_{2}$ occurred upon heating these crystals in air with excess $\mathrm{HgBr}_{2}$ gas at $230{ }^{\circ} \mathrm{C}$ for $16 \mathrm{~h}$ and $\mathrm{x}$-ray diffraction confirmed the $c$-axis lattice constant increased from 15.31 to $21.51 \AA$, as found previously. ${ }^{36}$ The intercalated crystals exhibited $T_{\mathrm{c}} \sim 74 \mathrm{~K}$ from magnetization and $\Delta \sim 24 \mathrm{meV}$ from MCT, indicating they are likely overdoped. ${ }^{24}$ Intercala-

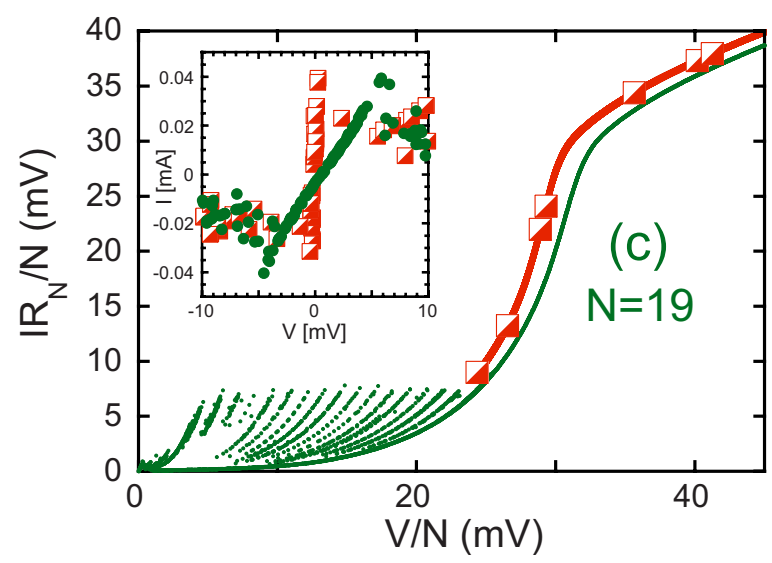

FIG. 3. (Color online) Details of curve (c) of Fig. 1 showing all quasiparticle branches and the $I(V)$ obtained after subtracting the initial SIN junction (half-open symbols). Inset: shows this subtraction corrects for the finite slope of the zero-voltage dc Josephson branch.

tion of $\mathrm{HgBr}_{2}$ between the $\mathrm{BiO}$ layers reduces the specific dissipation at fixed voltage by thickening the $\mathrm{Bi}_{2} \mathrm{Sr}_{2} \mathrm{O}_{4}$ tunnel barrier to obtain an order-of-magnitude decrease in the $c$-axis conductance. We can fine tune self-heating by a single control parameter, the stack height, since total heating power is proportional to $N$ for a constant mesa area.

Intercalated crystals were cleaved, sputter coated with gold and Ar-ion beam etched ${ }^{37}$ into arrays of $10 \times 10 \mu \mathrm{m}^{2}$ mesas using photolithography. Our MCT apparatus, described in Ref. 38, is also used to contact the gold film atop the mesa with a soft, $100-\mu \mathrm{m}$-diameter gold wire that is bent in a hook shape to minimize the contact force and any potential damage to the mesa (see inset of Fig. 1). This wire is sharpened to a diameter of 5-10 $\mu \mathrm{m}$ at the end touching the mesa and invariably the tip contacted a single mesa of the array. The multiple sweep $I(V)$ in Fig. 3 shows the Josephson current and the number of quasiparticle branches ${ }^{39}$ corresponding to the number, $N$, of IJJs in the mesa.

Single-junction methods were employed for comparison with the IJJ spectra. Both superconductor-insulator-normal (SIN) metal junctions and SIS break junctions were obtained on an intercalated crystal by MCT using a much thicker gold wire with a blunt tip. After collecting SIN data (e.g., Fig. 4) a hard contact is used to microcleave the underlying crystal leaving a chip of intercalated Bi2212 on the Au tip for subsequent SIS junctions, ${ }^{24}$ as shown in Fig. 1(d). Since heating is virtually eliminated for MCT, these data provide the equilibrium properties for interpreting the Bi2212 mesa data.

\section{EXPERIMENTAL RESULTS AND DISCUSSION}

The principal experimental data have been shown in Figs. 1 and 2 for the variable height mesas and MCT on intercalated Bi2212. It was necessary to account for the mesa's top contact that forms an SIN junction between the gold film and topmost $\mathrm{Cu}-\mathrm{O}$ bilayer that is in series with the IJJ stack. This is seen as a finite resistance (slope) for the zero-bias Josephson supercurrent (solid symbols of the inset in Fig. 3) that 


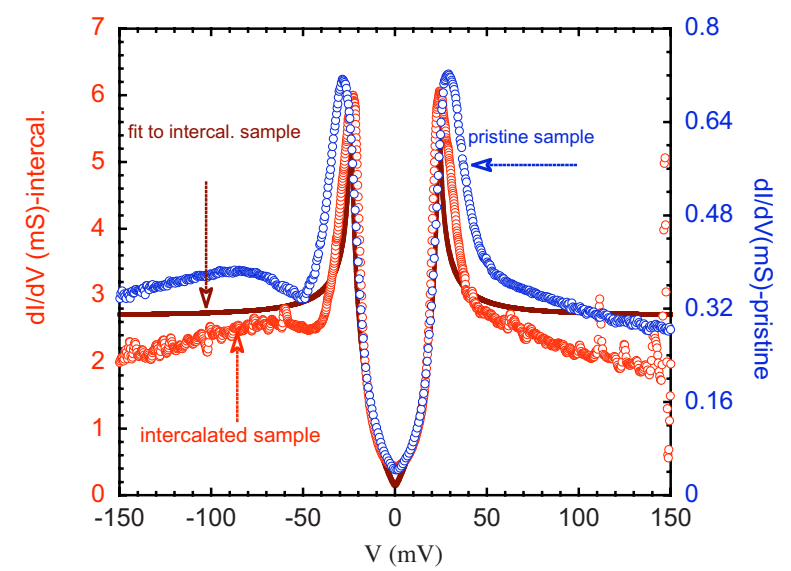

FIG. 4. (Color online) The $d I / d V$ from MCT for intercalated (solid diamonds) and pristine (open circles) Bi2212 are very similar and for subgap voltages they fit the momentum-averaged (Ref. 24) $d$-wave BCS model (Ref. 35) (solid line). Data are taken at $T_{\mathrm{B}}$ $\sim 4.2 \mathrm{~K}$. Above $\Delta$, the energy dependence of the normal-state density of states is clearly seen.

decreases with increasing $T$ in a manner that is only consistent with an SIN junction. To correct for this, our $I(V)$ from MCT in the SIN configuration (Fig. 4) is used to subtract the top-contact SIN voltage from the measured total voltage, for every current. The result (open symbols in both Fig. 3 and inset) is the $I(V)$ of the IJJ SIS stack alone and a numerical derivative generates the $d I / d V$ of Fig. 2. The biggest SIN correction yields a $10 \%$ lower peak voltage but our qualitative conclusions would be unaltered by neglecting this correction.

As pointed out in Sec. I, there is an excellent agreement among data from our MCT technique, STS, and ARPES with no significant discrepancies. ${ }^{23,32}$ Importantly, MCT and STS significantly reduce heating as these junctions exhibit much lower dissipation and better cooling than mesas. Our smallest mesa $(N=6)$ data in Fig. 2 look similar to that of these other techniques, including the DHF. The DHF is universally found in all previous MCT, ${ }^{19-25}$ STS, ${ }^{27-31}$ and angle-resolved photoemission $^{26}$ studies as well as IJJ data ${ }^{3,7,13}$ with significantly reduced heating. The telltale signs that the $d I / d V$ of Fig. 2 for $N \geq 12$ do not represent equilibrium at $T_{\mathrm{B}}$, even in the absence of backbending in $I(V)$, are (1) anomalously high and narrow peaks in the taller mesas compared to MCT and STS data; (2) significantly reduced peak voltages from the MCT value; and (3) the absence of DHF.

To validate the generality of our results, we note that intercalation of $\mathrm{HgBr}_{2}$ has only a small effect on the DOS of the $\mathrm{Cu}-\mathrm{O}$ bilayers as revealed by the similarity of the SIN data in Fig. 4, with and without intercalation. The subgap conductance for both curves is well modeled by the $d$-wave DOS of Eq. (1) and the fit, shown only for the intercalated crystal, gives $\Delta_{0}=24 \mathrm{meV}$ and $\Gamma=0.55 \mathrm{meV}$ while the oxygen overdoped pristine Bi2212 crystal fit (not shown) yields $\Delta_{0}=26 \mathrm{meV}$ and $\Gamma=0.6 \mathrm{meV}$, and both use the same momentum-averaging parameter. ${ }^{24}$ At higher voltages, Eq. (1) is inadequate as it misses the actual normal-state conductance and the $E$ dependence of $\Delta$ : the latter produces the DHF (Ref. 32) that is seen more prominently at negative voltages in Fig. 4.
The tunneling conductance of an SIS MCT break junction in Fig. 2 exhibits both Josephson (small peak at zero bias) and quasiparticle tunneling. For SIS break junctions at $4.2 \mathrm{~K}$, their conductance peak voltages are an excellent measure of $2 \Delta .{ }^{20,24}$ Thus $\Delta=24 \mathrm{meV}$ from the SIS peaks matches that of the SIN junction fit of Fig. 4. The dip feature located at 80 $\mathrm{mV}$ is more readily visible in the SIS data but can be traced consistently $^{23}$ to the feature at $-50 \mathrm{mV}$ in the SIN DOS of Fig. 4. For example, using the difference between the dip minimum and the peak voltage as a crude estimate of the strong-coupling boson energy, ${ }^{22}$ this gives approximately 25 $\mathrm{meV}$ and $30 \mathrm{meV}$ for the SIN and SIS junctions respectively, a reasonable consistency. Notably, data well above the peaks coincide for all mesas with $N=12-30$ in Fig. 2, implying that all vestiges of superconductivity are gone. Then $N d I / d V$ at the dip/hump for $N=6$ is clearly seen to fall below/above that normal-state value as expected for strong-coupling selfenergy effects linked to superconductivity, and in agreement with the MCT data. ${ }^{19-25}$

While the conductance peaks in Fig. 2 for mesas with $N>6$ are reminiscent of the superconducting coherence peak at $2 \Delta$, that interpretation is incorrect. First, the peak is at a voltage that is significantly smaller than $2 \Delta / e$, the equilibrium value from MCT. Furthermore, as the mesa height, and thus dissipation, increases, the peak voltage per junction decreases and that trend is suggestive of heating. Note that the ratio, $\beta$, of a peak's full width half maximum to its voltage (inset of Fig. 2), is a small, almost constant value for $N$ $=12-30$ while it is considerably larger for the $N=6$ mesa and the MCT data that both exhibit a well-defined DHF. The data reveal an abrupt change in $\beta$ by nearly a factor of 6 occurring between $N=6$ and 12. This large change in $\beta$ signals an important crossover from near-to-equilibrium superconducting properties to severe self-heating. To make this result more general, the inset of Fig. 2 includes data from Yurgens et al. ${ }^{3}$ and Zhu et al. ${ }^{7}$ which are on mesas with $N=10-11$. The Zhu data are particularly useful as they utilize a different independent control of self-heating via the mesa area. Nevertheless, a similar abrupt change in $\beta$ versus mesa area is observed in the inset of Fig. 2, and it implies that smaller area mesas better utilize the lateral spread of heat in the underlying crystal (suggested in Ref. 15). For $\beta>0.3$, both the Yurgens and Zhu data observe the characteristic DHF but for $\beta<0.15$ this feature disappears.

Note that recent STS data ${ }^{30}$ show that the DHF disappears as $T$ approaches $T_{\mathrm{c}}$ so its absence for $N=12-30$ in Fig. 2 likely indicates that $T_{\mathrm{m}}>T_{\mathrm{c}}$ after the peak value of $\mathrm{NdI} / \mathrm{dV}$ drops down to its normal-state value. In addition, this onset of normal-state behavior corresponds to the same dissipated power, of $1-1.1 \mathrm{~mW}$, for $N=12-30$. For $N=6$, the DHF occurs at power levels between $\sim 0.5$ and $1 \mathrm{~mW}$ while for $N$ $=12$ a very faint feature can still be seen at $\sim 0.9 \mathrm{~mW}$.

To summarize this section, we have shown that sharp conductance peaks $(\beta<0.15)$ in taller mesas are not a measure of $\Delta(T)$ despite their gaplike appearance. Rather, their origins are the sharp rise in $I(V)$ that represents transitions of the mesa into the normal state, and these are found systematically over a range of $N$. Having established these points experimentally, the following section investigates several heating scenarios and find substantive agreement with a 
readily justifiable lateral temperature gradient in the mesa as the cause of these $I(V)$.

\section{HEATING MODELS}

The electrical power dissipated within the tunnel junctions of a mesa results in an increase in the average excitation energies of the electron and phonon systems. The short relaxation times found at temperatures of 50-100 K allow one to define an effective mesa temperature, $T_{\mathrm{m}}$, for these steady-state electron and phonon distribution functions. It has been shown ${ }^{16,17}$ that Newton's law of cooling, ${ }^{17}$

$$
T_{\mathrm{m}}=T_{\mathrm{B}}+\alpha P,
$$

is a good approximation for $\mathrm{Bi} 2212$ mesas. Here $P=I V, V$ is the voltage across the stack of SIS junctions, $\alpha$ is the effective thermal resistance, and $T_{\mathrm{B}}$ is the bath temperature. For spatially uniform heating, one would always probe a near-toequilibrium system that would be, however, at a current (or power) dependent $T_{\mathrm{m}}$ given by Eq. (2).

Existing heating models typically result in backbending of the $I(V)$, such as Figs. 1(a) and 1(b), whereas there is a ubiquitous lack of backbending in our mesa data reported here for intercalated Bi2212 [e.g., Fig. 1(c)] and previous work $^{8-12}$ on pristine Bi2212. Initially, we address and dismiss two potential heating explanations for the lack of backbending. First, we consider whether the severe intrinsic broadening of the DOS as $T_{\mathrm{c}}$ is approached could smear out the $I(V)$ enough to eliminate type-II backbending in a uniform self-heating scenario. Further below, we consider the possibility that $\rho_{\mathrm{c}}(T)$ for intercalated Bi2212 has a weaker temperature dependence than pristine $\mathrm{Bi} 2212$ so that the heating-induced type-I backbending ${ }^{16}$ would not occur. Finally, we outline a model of strong self-heating with a laterally nonuniform mesa temperature, and show that it can recover the measured nonbackbending $I(V)$ with a conductance peak that sharpens and moves to lower voltage for stronger heating (experimentally emulated by increasing only the stack height, $N$, as in Fig. 2).

To address severe intrinsic broadening, we note that for Bi2212, several factors could reduce the likelihood of observing type-II backbending. The $d$-wave DOS [Eq. (1)] does not produce as sharp a jump in $I(V)$ at $V=2 \Delta$ as $s$-wave superconductors, even when the smearing $\Gamma$ is zero. Considering the actual $\Gamma$ is large, and that it increases dramatically as $T_{\mathrm{c}}$ is approached, this effect might eliminate type-II backbending even for strong heating and thus reproduce our mesa data. We test this possibility by calculating the $I(V)$ for uniform heating in Bi2212 mesas using experimental STS data for the temperature dependencies of $\Delta(T)$ and $\Gamma(T)$ in Eq. (1). However, the result of the following calculation always produces type-II backbending for $N>12$.

Even for spatially uniform heating, the $I(V)$ would not represent any constant-temperature equilibrium $I_{\mathrm{eq}}(V, T)$ found with negligible dissipation (e.g., by MCT). To emulate our mesa $I(V)$ data, we need to self-consistently determine for each $I$ and $V$ an effective $T_{\mathrm{m}}$, for which $I, V$, and $T_{\mathrm{m}}$ satisfy both Eq. (2) and the equilibrium $I_{\text {eq }}\left(V, T_{\mathrm{m}}\right)$. Thus we determine the mesa $I(V)$ for a stack of $N$ junctions by a

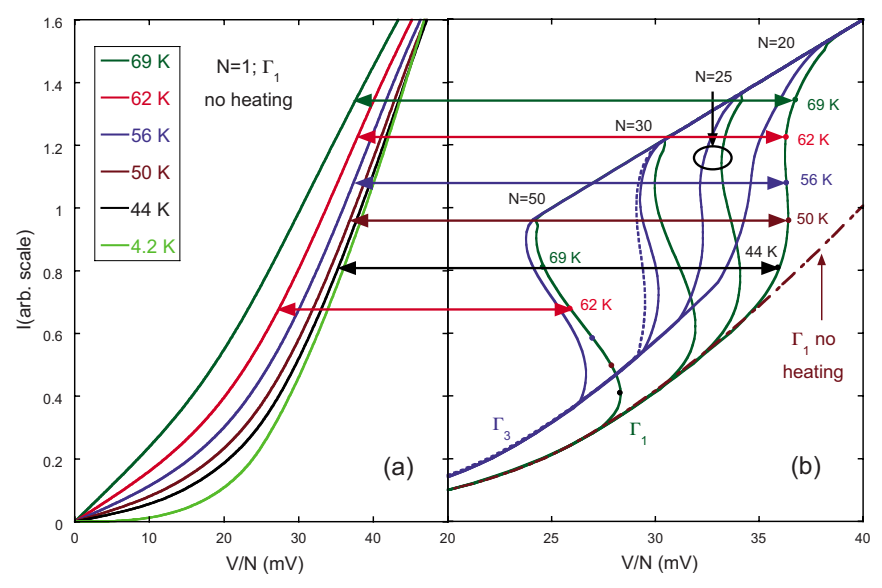

FIG. 5. (Color online) Numerical simulations of $I(V)$ using $\Delta(T)$ and $\Gamma(T)$ from reported fits to the experimental STS data (Ref. 31). (a) Equilibrium $I(V)$ for various fixed temperatures $T$, using $\Delta_{1}$ (low-temperature gap of $22 \mathrm{meV}$ ) and $\Gamma_{1}$ from Ref. 31. (b) Uniform-temperature, self-heating $I(V)$ for various $N$ and two of the data sets $\Delta_{1}, \Gamma_{1}$ and $\Delta_{3}, \Gamma_{3}$ taken from Ref. 31 (calculations for $\Delta_{2}$, $\Gamma_{2}$ lie between these) using $\alpha=70 \mathrm{~K} / \mathrm{mW}$ and $T_{\mathrm{B}}=4.2 \mathrm{~K}$. Dashed curve for $N=19$ shows effect of doubling the quasiparticle lifetime smearing, i.e., $\Gamma=2 \Gamma_{3}$. For two curves, the local uniform $T_{\mathrm{m}}$ values are shown and they correspond to points on the equilibrium $I(V)$. This correspondence was used in Refs. 16 and 18 but in those cases only the linear, low-voltage portion of the equilibrium $I(V)$ were needed, e.g., Fig. 1(a). See text for further details.

straightforward solution of these two independent relations among the values of $I, V$, and $T_{\mathrm{m}}$.

We generate equilibrium $I_{\text {eq }}(V, T)$ from standard tunneling theory of SIS junctions using the DOS of Eq. (1) and experimental values of $\Delta(T)$ and $\Gamma(T)$ from STS data. ${ }^{31}$ These STS data are similar to the MCT example of Fig. 4 but over a wide range of $T$. That study ${ }^{31}$ used one pristine Bi2212 crystal (bulk $T_{\mathrm{c}} \sim 74 \mathrm{~K}$ ) and found numerous data sets that were ascribed to a variation in the local properties across the crystal surface. We used three of these data sets, labeled according to their low-temperature gaps, $\Delta_{1}, \Delta_{2}$, and $\Delta_{3}=22 \mathrm{meV}$, $23 \mathrm{meV}$, and $25 \mathrm{meV}$, respectively. These gaps and $T_{\mathrm{c}}$ are close to those reported here, and thus should be a good approximation to our intercalated crystals [recall that Fig. 4 shows the similarity of $N(E)$ for pristine and intercalated Bi2212 MCT data]. Each STS data set, $\Delta(T)$ and $\Gamma(T)$, generates the quasiparticle DOS from Eq. (1), which is then convoluted with itself to predict the equilibrium $I_{\mathrm{eq}}(V, T)$, that are shown in Fig. 5(a) for STS data set $\Delta_{1}, \Gamma_{1}$.

To illustrate the effects of self-heating, we consider a fixed current, I. Then the allowed mesa voltages are represented by two independent, single-valued functions for each fixed $I$ : (1) $V_{1}\left(I, T_{\mathrm{m}}\right)=N V_{\mathrm{eq}}\left(I, T_{\mathrm{m}}\right)$, where $V_{\mathrm{eq}}\left(I, T_{\mathrm{m}}\right)$ is the inversion of $I_{\mathrm{eq}}\left(V, T_{\mathrm{m}}\right)$ and it is generally a decreasing function of $T_{\mathrm{m}}$; and (2) $V_{2}\left(I, T_{\mathrm{m}}\right)=\left(T_{\mathrm{m}}-T_{\mathrm{B}}\right) / \alpha I$, which follows from Eq. (2) and is an increasing function of $T_{\mathrm{m}}$. The intersection of the two functions is the self-consistent solution for the mesa voltage, $V$, and temperature, $T_{\mathrm{m}}$, for each value of $I$. This procedure, repeated for all values of $I$, leads to the full $I(V)$, shown in Fig. 5(b) for $N=20-50$ using the data sets of Ref. 31 (labeled $\Gamma_{1}$ and $\Gamma_{3}$ while $\Gamma_{2}$ data, not shown, fall 
between them). Here we use $\alpha=70 \mathrm{~K} / \mathrm{mW}$ since then the experimental data for each of the mesas for $N=12-30$ would reach $T_{\mathrm{c}} \sim 74 \mathrm{~K}$ as the $d I / d V$ of Fig. 2 drop to the normalstate value. Although these solutions represent spatially uniform heating, the effective $T_{\mathrm{m}}$ will be current (or power) dependent, and representative $T_{\mathrm{m}}$ values are shown on a few of the curves in Fig. 5(b).

That all data sets for $N>20$ show backbending implies that the strong quasiparticle damping, $\Gamma(T)$, near $T_{\mathrm{c}}$ is insufficient to smear it out. To directly test the effect of $\Gamma$, a third dashed curve is shown in Fig. 5(b) for $N=30$ that uses $\Gamma(T)=2 \Gamma_{3}(T)$ and the degree of backbending is reduced but not eliminated. For the $N=20$ mesa calculation, backbending is avoided for $\alpha \leq 70 \mathrm{~K} / \mathrm{mW}$, and that crossover $\alpha$ value will scale as $1 / N$. Thus while a small enough $\alpha$ would obviously emulate the negligible heating of our MCT data and eliminate backbending, the conductance peaks would be broader and bunched together at voltages much closer to $2 \Delta$ than observed in our mesa data. As the backbending observed in these calculations is totally absent in all experimental $I(V)$, the uniform heating model is inadequate.

To clarify this conclusion, note that for $\alpha=70 \mathrm{~K} / \mathrm{mW}$, the $N=20$ mesa could exhibit a sharp conductance peak in the uniform heating model of Fig. 5(b) but with that $\alpha$, the $N$ $=12$ peak would be significantly broader and $N=30$ would exhibit backbending. To maintain the same peak sharpness for $N=12$ and 30 would require a variation in $\alpha$ with $N$ (power) that is unphysical, i.e., $\alpha \sim 110 \mathrm{~K} / \mathrm{mW}$ for the $N$ $=12$ mesa and $\alpha \sim 43 \mathrm{~K} / \mathrm{mW}$ for the $N=30$ mesa. In addition, under this scenario, the $N=30$ mesa would achieve $T_{\mathrm{c}}$ at $V / N \sim 36 \mathrm{mV}$ that is well above the conductance peak at $V / N \sim 26 \mathrm{mV}$, yet there is no evidence of a dip feature in this data. Whereas the $N=12$ mesa would already achieve $T_{\text {c }}$ when the conductance peak drops to half of its maximum but in this case there is evidence of a weak dip feature above this point. These observations underscore the inability of a uniform heating model to explain our observation of sharp peaks of roughly constant width for a wide range of $N$ values (as are shown in Fig. 2). The potential instability of intrinsic backbending into two-phase coexistence might rectify this discrepancy ${ }^{40}$ but we will show below that a physically sensible lateral temperature gradient (due to the Au-tip contact), also accomplishes this.

We now consider and dismiss a second potential heating explanation of our data: whether the particular $\rho_{\mathrm{c}}(T)$ for intercalated Bi2212 could eliminate type-I backbending. There is one set of experimental $\rho_{\mathrm{c}}(T)$ data for intercalated Bi2212 that is known to us and it reports data as a function of applied magnetic field parallel to the $c$ axis. ${ }^{33}$ It has been used to generate the low-voltage $I(V)$ using Newton's law of cooling [Eq. (2)] to obtain the (assumed uniform) mesa temperature, and for fields of 4 and $14 \mathrm{~T}$, type-I backbending was not found. ${ }^{17}$ However, our analysis of the $1 \mathrm{~T}$ data of Ref. 33 clearly shows type-I backbending would be expected; so suggesting its absence in zero field is problematic. An additional issue with this scenario is that the voltages of our sharp peaks are fairly close to $2 \Delta\left(T_{\mathrm{B}}\right) / e$ so one would not anticipate type-I backbending to dominate, even if it did occur for stronger heating at lower voltages.

Finally, we present a heating scenario that does explain our data. In order to explain the very narrow peaks and ab- sence of backbending, we explore the possibility of a transition to the normal state via coexistence of two phases within the mesa area. One part of the mesa area could exhibit a smaller-than-average current density and power dissipation to remain superconducting $\left(T<T_{\mathrm{c}}\right)$ while another portion, a hot spot, supports the normal state $\left(T_{\mathrm{hs}} \geq T_{\mathrm{c}}\right)$ with a compensating larger-than-average current (power) density. While this could occur ${ }^{40}$ via instability of the intrinsic S-shaped $I(V)$ calculated above for uniform heating, we believe it is more likely here to be due to the asymmetrical cooling caused by our Au tip contacting the top of the mesa.

Two-phase coexistence is not new. A sharp conductance peak was previously documented in low $-T_{\mathrm{c}}$ superconducting junctions ${ }^{40}$ resulting from a spontaneous instability into an inhomogeneous current density that induced predominantly a nonequilibrium state of smaller $\Delta$ rather than simple heating. The occurrence of hot spots $\left(T_{\mathrm{hs}} \varepsilon T_{\mathrm{c}}\right)$ with a size proportional to the current has been reported recently in very large Bi2212 mesas. ${ }^{41}$ However, unlike the present data, these cases exhibit a jump in the $I(V)$ to lower voltage and higher current as an initial hot spot nucleates. Rationalizing this difference requires a digression into the thermal boundary conditions for various mesa configurations. That an increase in current leads to a larger hot spot is an integral part of any two-phase model and is justified by the data of Refs. 40 and 41 . The relevant point is whether the mesa voltage increases or decreases as the hot spot expands.

The data of Zhu et al., ${ }^{7}$ who varied only the mesa area, provide the first insight (see Fig. 2 inset). Decreasing the area led to less heating, and eventually, for mesa areas $<1 \mu \mathrm{m}^{2}$, they found broad conductance peaks and close-toequilibrium properties. This is most readily explained by a more effective lateral transport of heat, in the mesa and the nearby regions of the underlying crystal, as the mesa area decreases. Such an idea was also expressed in Ref. 15. To see this, consider heat removal from the central region of a very large mesa: it will be virtually one dimensional (along the $c$ axis of the underlying crystal). On the other hand, very small mesas will enjoy three-dimensional cooling into the full $2 \pi$ steradians of the underlying crystal.

Translating this idea into a two-phase model, larger hot spots exhibit poorer cooling and thus require less power density to maintain $T_{\mathrm{hs}} \geqslant T_{\mathrm{c}}$. The largest hot spot would be the entire mesa area, and that is represented by the (highest) normal-state curve in Fig. 5(b), for which the calculated temperatures are $\geqslant T_{\mathrm{c}}$. For mesas biased by a low-impedance (voltage) source, an abrupt global transition of the entire mesa would be expected. For the more usual experimental case of higher-impedance sources, the transition would be a jump along the electrical load line that could end up at an intermediate sized hot spot, with lower voltage and higher current. The occurrence of the jump and its location on the $I(V)$ are dictated by the condition that the power density in the hot spot, $(V / t)^{2} / \rho_{\mathrm{cNc}}$, after the jump is sufficient to raise $T$ to $T_{\mathrm{c}}$. Here $t$ and $\rho_{\mathrm{cNc}}$, the mesa height and normal-state $c$-axis resistivity at $T_{\mathrm{c}}$, respectively, are virtually constant. This scenario is likely the explanation of the jumps seen in the large mesa ${ }^{41}$ and our Fig. 1(b). Note that the entire type-I backbending $I(V)$ would be experimentally observable with a sufficiently high-impedance, constant-current bias but for 
large area mesas, it should be noted that the remainder of the mesa acts to voltage bias the hot-spot region. ${ }^{40}$

For the intercalated mesas reported here, heat removal through the underlying crystal is more problematic. The large $\mathrm{HgBr}_{2}$ intercalant molecules should behave as phonon rattlers $^{42}$ to reduce the thermal conductivity compared to pristine mesas (e.g., 30 times smaller thermal conductivity was found with phonon rattlers in Ref. 42). As such, the relatively low thermal resistance of our 100 - $\mu \mathrm{m}$-diameter, $\sim 0.5$-cm-long $\mathrm{Au}$ wire $(\sim 2 \mathrm{~K} / \mathrm{mW})$ contacting the $40 \mathrm{~nm}$ $\mathrm{Au}$ film atop our mesas (Fig. 1 inset) becomes a more significant heat sink. If dominant, this Au wire would critically alter the heat-flow path from a situation in which the underlying crystal is the main heat sink. Then the hot spot should nucleate farthest from the (random) position of the Au-wire contact. Another way to visualize this continuous transition to the normal state is to recognize that the Au-wire heat sink produces a thermal gradient across the mesa whenever power is dissipated. The most remote points will reach $T_{\mathrm{c}}$ first and that phase boundary will move continuously toward the heat sink as the current (power) is increased. As mentioned above, the hot spot size will increase as the current increases so our task is to evaluate whether the voltage increases or not.

A precise calculation is out of our reach since the exact location of the Au tip and its contact area are not knowable. However, the ubiquitous nature of the sharp peak for various $N$ values and subsequent contacts in new locations on these mesa arrays imply that the precise details are unimportant. Thus two one-dimensional (1D) approximations will be considered here: (1) the mesa is divided into cells along a 1D temperature gradient of length $5 \mu \mathrm{m}$; and (2) the mesa is divided into concentric rings with a 0.5 - $\mu \mathrm{m}$-diameter cold $\mathrm{Au}$ tip at the center. These geometries represent tractable models for two extremes: (2) the Au tip is at the center of the mesa and (1) the Au tip near the edge. In each case we numerically integrate the steady-state heat-flow equation from the hottest end, with temperature $T(1)$, to the Au tip that is tied to the bath temperature, $T_{\mathrm{B}}$. The decrease in $T(m)$ in going to the next cell is just

$$
T(m+1) \sim T(m)-P(m) \delta x / \kappa_{\mathrm{ab}}[T(m)] A,
$$

where $P(m)$ is the sum of $V(m) I(m)$ for all cells up to $m, \delta x$ is the cell spacing along the gradient, $\kappa_{\mathrm{ab}}[T(m)]$ is the $a b$-plane thermal conductivity, and $A$ is the cross sectional area for the heat current. To find the local power dissipation, $P(m)$, we first note that the Au film atop the mesa and the high transport anisotropy of $\mathrm{Bi} 2212$ assures a uniform $V$ across the entire mesa area, even with a temperature gradient and/or two-phase coexistence. Then for a specific $V$, the local current, $I(m)$, in a given cell, $n$, is calculated from the equilibrium $I_{\text {eq }}[V, T(m)]$, shown in Fig. 5(a), the cell area and its local temperature, $T(m)$. Now $A=w c_{\text {eff }}$ where $w$ in pure 1D problem (1) is the fictitious width that cancels out while it is the circumference of the $m$ th ring in (2), and $c_{\text {eff }}$ accounts for the mesa height and the depth into the underlying crystal involved in heat flow from the hot end to the Au tip. The anisotropy of thermal conductivity in Bi2212 is not too large $\left(\kappa_{\mathrm{ab}} \sim 6 \kappa_{\mathrm{c}}\right)$ so $c_{\text {eff }}$ could be a reasonable fraction of the

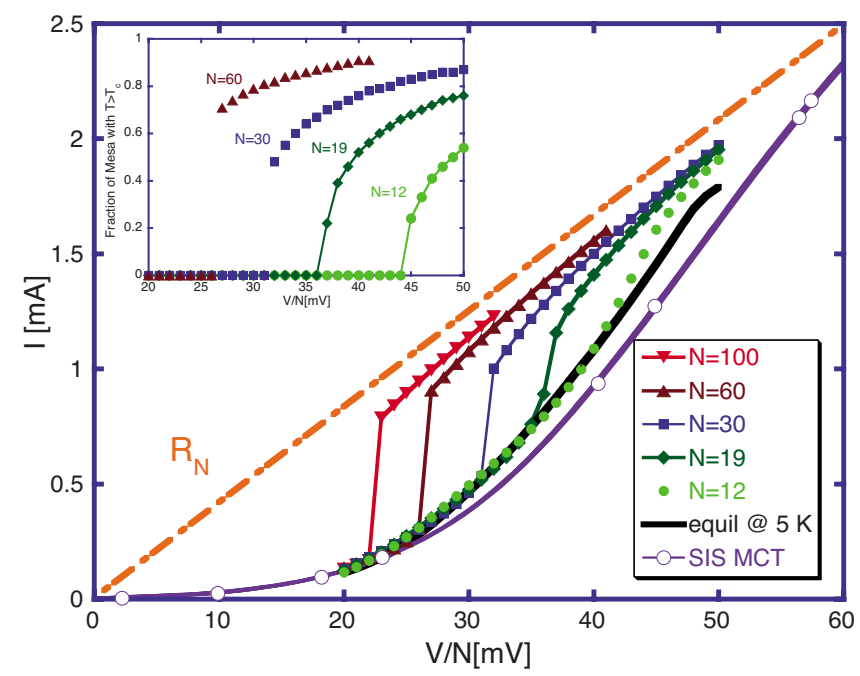

FIG. 6. (Color online) Results of 1D-heating calculation (concentric annuli) display the qualitative features of mesa data (Figs. 1 and 2) as a function of mesa height $(N)$. The width of the transition narrows and shifts to lower voltage as $N$ increases. The equilibrium $I(V)$ at $5 \mathrm{~K}$ for the DOS used in the calculation is shown as the dark, thick solid line. It differs importantly in its steepness compared to the $I(V)$ at $\sim 5 \mathrm{~K}$ measured by MCT (line with open circles) on an intercalated Bi2212 crystal equivalent to the ones used for mesas. All low-temperature $2 \Delta / e$ are scaled to $48 \mathrm{meV}$. The inset shows the fraction of the 1D length that has $T>T_{\mathrm{c}}$ for four of the calculations.

10 - $\mu \mathrm{m}$-mesa width rather than the much smaller mesa height (13-65 nm).

To apply the boundary conditions, we note that for given $V, N, \kappa_{\mathrm{ab}}$, and $c_{\text {eff }}$ there will be a unique value of $T(1)$, found by iteration, for which the final $T\left(m_{\mathrm{f}}\right)=T_{\mathrm{B}}$. The total mesa current for this solution is just the sum of $I(m)$ up to $m_{\mathrm{f}}$, and in this way the $I(V)$ and the temperature profile are calculated. The dependence on $N$ comes solely from the proportionality of $P(m)$ to $N$. These results are displayed in Fig. 6 for approximation (2), where we use a half of the measured $\kappa_{\mathrm{ab}}$ of pristine $\mathrm{Bi} 2212$ and $c_{\text {eff }}=1 \mu \mathrm{m}$. We used 100 cells along the 1D thermal gradient: the difference using 200 cells was less than the size of the symbols, which is certainly smaller than the absolute accuracy that can be expected from these approximate 1D models. Note also that since $T$ is nonuniform, no effective $\alpha$ value can be ascertained.

These calculated $I(V)$ imply a conductance peak that sharpens and moves to lower voltage for larger $N$, i.e., stronger heating (experimentally emulated by increasing only the stack height, $N$, as in the data of Fig. 2). That qualitative agreement with our data upon using reasonable parameters indicates that this scenario might be closer to the actual situation than the constant $T$ calculation of Fig. 5. The inset of Fig. 6 shows that for $N \geq 30$, the fraction of mesa with $T$ $>T_{\mathrm{c}}$ jumps from zero to $\sim 45 \%$ at the jump in $I(V)$. The first normal region occurs at $V / N \sim 37 \mathrm{mV}$ for $N=19$ while for $N=12$, it is at $V / N \sim 45 \mathrm{mV}$, and these rise gradually to $55-75 \%$ for the highest $V / N$ shown.

However, we need to discuss the jump in current at lower voltages $(V / N)$ for larger $N$. Such jumps would be expected if backbending occurs in the full $I(V)$, which, however, can- 
not be calculated by the above approach. The crossover voltage from a smooth $I(V)$ to a jump, at $V / N \sim 30 \mathrm{mV}$, is virtually independent of (a) the two approximations (1) and (2); (b) the parameter choices for $N, \kappa_{\mathrm{ab}}, c_{\text {eff }}$, etc.; (c) the inclusion of a boundary resistance at the Au-tip contact; or (d) the inclusion of heat flow out of the mesa through the substrate. For (b), this is at least partially due to the clear interaction between some parameters, e.g., $\kappa_{\mathrm{ab}}$ and $c_{\text {eff }}$ always appear as their product and increasing $\kappa_{\mathrm{ab}}$ has a similar effect as decreasing $N$. However, we are unable to find conditions that bring this crossover to below the measured peak at $V / N$ $\sim 26 \mathrm{mV}$ for $N=30$.

The intransigence of this crossover $V / N$ to significant changes in the heating model implies it does not result from specific details of our heating model, and instead might be caused by the specific intrinsic properties of the DOS. Our $1 \mathrm{D}$ heating models use the $d$-wave DOS [Eq. (1)] with $\Delta(T)$ and $\Gamma(T)$ from STS data on pristine Bi2212. This issue can be addressed by comparing the calculated $I(V)$, without heating, to the experimental MCT data for intercalated Bi2212. Such a comparison is shown in Fig. 6 at intermediate voltages and there are clear differences. This disagreement could result if either the $d$-wave DOS [Eq. (1)] or $\Delta(T)$ or $\Gamma(T)$ values taken from STS data on pristine Bi2212 are an inadequate approximation for intercalated $\mathrm{Bi} 2212$. In particular, the calculated $I(V)$ is steeper in the region of the instability than the intercalated $I(V)$ data. It is not difficult to imagine that this steeper behavior would be more prone to runaway heating and instability. High-temperature $I(V)$ data on intercalated Bi2212 would overcome this deficiency but it has not been possible due to mechanical instabilities caused by differential thermal expansion in the MCT apparatus.

This previous discussion may help explain why in data in Fig. 1(b) for the pristine Bi2212 mesa with the same area and $N=28$ behave so differently. In curve (b), there is a jump seen for $I R_{\mathrm{N}} / N \sim 3.9 \mathrm{mV}$ announcing the abrupt formation of a finite-size hot $\operatorname{spot}^{41}$ while the backbending part of the curve may be of type-I and effectively explained by the temperature dependence of $\rho_{\mathrm{cN}}\left(T_{\mathrm{m}}\right)$ in the regions outside the hot spot. ${ }^{16}$ The pristine, unintercalated Bi2212 mesa in curve (b) exhibits an order-of-magnitude larger power density, for a given voltage per junction, but more importantly, as mentioned above, a higher thermal conductivity for the mesa and its underlying crystal than for the intercalated mesa (c). Therefore for the pristine Bi2212 mesa, curve (b), the thermal agenda may be set more by the underlying crystal than the gold-point top contact. Thus it appears that subtle differences in the thermal boundary conditions can tip the balance between abrupt hot-spot formation and a continuous lateral thermal gradient.

To address this, we note that for the $N=28$ pristine mesa of curve (b), the heat-transfer coefficient is found to be $\alpha$ $=38 \mathrm{~K} / \mathrm{mW}$, by using the methods of Ref. 16 with the full set of $I(V, T)$, whereas $\alpha=70 \mathrm{~K} / \mathrm{mW}$ for the intercalated mesas (see above). The main heat-flow difference between these is the thermal conductivity of the underlying crystal. The lower thermal resistance for the pristine mesa implies that the underlying crystal is a more important source of cooling, and thus its behavior may not so closely emulate the above thermal-gradient heating calculation.

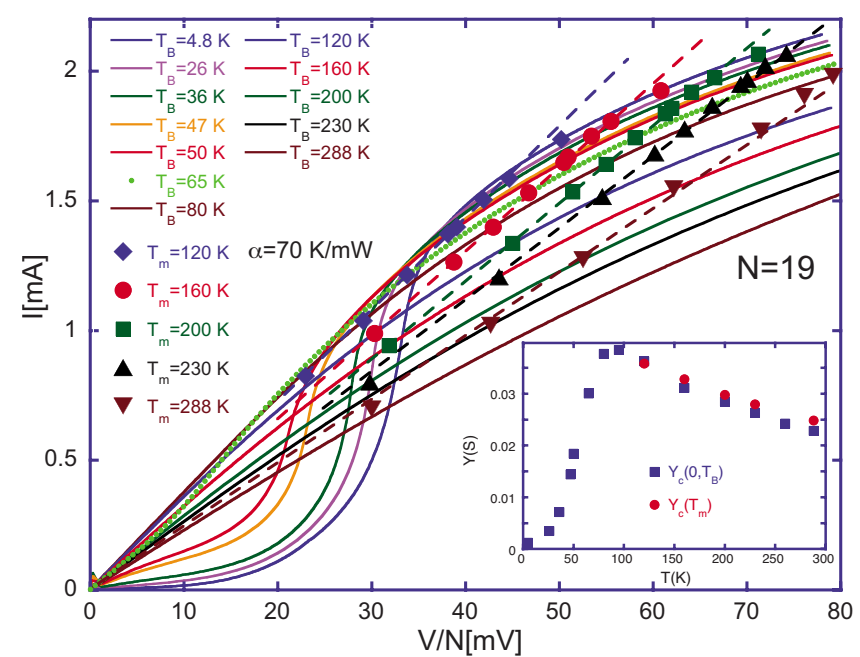

FIG. 7. (Color online) The bath temperature, $T_{\mathrm{B}}$, dependence of the experimental $I(V)$ for the $N=19$ intercalated mesa. The gaplike feature at $\sim 33 \mathrm{mV}$ for $T_{\mathrm{B}}=4.8 \mathrm{~K}$ is seen to decrease with increasing $T_{\mathrm{B}}$ and completely disappear for $T_{\mathrm{B}}=80 \mathrm{~K}$. Newton's law of cooling using $\alpha=70 \mathrm{~K} / \mathrm{mW}$ predict isothermal points on these curves for the indicated $T_{\mathrm{m}}$. The slight disagreement points on the uppermost curve $(4.8 \mathrm{~K})$ are consistent with a larger value of $\alpha$ at such a low temperature. The resulting linear isothermal conductance (dashed lines) implies no pseudogap for intercalated (overdoped) $\mathrm{Bi} 2212$, and the values agree with the zero-bias conductance at the same $T_{\mathrm{B}}$ (see inset: squares are zero bias $Y$ at $T_{\mathrm{B}}$; circles are slopes of dashed lines in main body of this figure).

To summarize, the variety of behavior seen in Bi2212 mesas (Fig. 1) may be understood within a heating model that includes two-phase coexistence for highly driven mesas and uniform heating for moderately driven mesas.

\section{TEMPERATURE DEPENDENCE}

Our data, modeling and discussion, so far, have concentrated on $T_{\mathrm{B}} \sim 4.2 \mathrm{~K} \ll T_{\mathrm{c}}$. Figure 7 shows the dependence of the experimental $I(V)$ on $T_{\mathrm{B}}$ for the mesa with $N=19$, for which we have already shown that its "gaplike" feature, shown for $4.2 \mathrm{~K}$ in Fig. 1(c), is consistent with a lateral thermal gradient in the mesa. Upon increasing $T_{\mathrm{B}}$, this feature shifts to lower voltages and disappears completely at $T$ $=80 \mathrm{~K}$ where the $I(V)$ shows no obvious gaplike features. The observed curvature for $V>2 \Delta$ can be entirely ascribed to a heating effect. To see this, we have used Eq. (2) with $\alpha=70 \mathrm{~K} / \mathrm{mW}$ to determine isothermal points on each curve of Fig. 7 for $T_{\mathrm{m}}=160 \mathrm{~K}$ (circles), $200 \mathrm{~K}$ (squares), $230 \mathrm{~K}$ (triangles), and $288 \mathrm{~K}$ (inverted triangles). At each $T_{\mathrm{m}}$, the linear conductance, $Y_{\mathrm{c}}\left(T_{\mathrm{m}}\right)$, closely mimics the zero-bias conductance, $Y_{\mathrm{c}}\left(0, T_{\mathrm{b}}\right)$, at the same temperatures (inset of Fig. 7). The slight discrepancy at the highest $T$ may be due to our use of a temperature-independent $\alpha$. This linear $Y_{\mathrm{c}}\left(T_{\mathrm{m}}\right)$ shows an absence of any pseudogap for intercalated (overdoped) Bi2212, and this is consistent with its absence in overdoped pristine Bi2212. ${ }^{21}$

A further conclusion is that the gaplike feature appears to close at $T_{\mathrm{C}}$ in the manner of the superconducting gap in 
conventional superconductors in equilibrium. However, such behavior is also expected from self-heating. As $T_{\mathrm{B}}$ increases, less voltage (heating power) is required to reach $T_{\mathrm{C}}$ and for $T_{\mathrm{B}}>T_{\mathrm{C}}$ the mesa is already in the normal state so no transition is possible. This discussion points out how misleading the heating effect can be. Not only does the sharp upturn in $I(V)$ mimic the expected behavior of a tunnel junction at the gap voltage but its disappearance above $T_{\mathrm{C}}$ seems to confirm (incorrectly) its assignment as a superconducting energy gap.

\section{SUMMARY AND CONCLUSION}

In summary, we have clearly identified a regime of strong self-heating in Bi2212 mesas which is characterized by the transition of the mesa into the normal state without backbending of the $I(V)$. At a voltage of this transition, $V$ $<\Delta\left(T_{\mathrm{B}}\right) / e$, the $I(V)$ exhibits a near vertical rise and consequently high, sharp conductance peaks which can easily be misinterpreted as superconducting energy gaps. While heating had been suggested previously, ${ }^{4,7,17}$ it was only partially documented without knowledge of the actual equilibrium gap value or the intrinsic width of the DOS. In addition, this was a counterintuitive suggestion, and its lack of universal recognition by the scientific community is evidenced by the ongoing publications. ${ }^{8-12}$ Our use of independent MCT junctions on the same (or similar) crystals has provided the key equilibrium data for comparison. It is found that a relatively broad peak and well-defined dip/hump features characterize the equilibrium SIS conductance, which is consistent with other single-junction tunneling studies of Bi2212. A reproducible gap value, $\Delta=24 \mathrm{meV}$, is obtained from both SIN and SIS MCT junctions, indicating these crystals are overdoped. ${ }^{24}$ Our smallest mesa with $N=6$ more closely resembles the MCT data, including a well-defined dip/hump and a broader conductance peak, and the latter systematically shifts to lower voltages and abruptly sharpen as $N$ increases. The demonstration of this evolution is one of the important findings of the present study. That the sharp peaks occur at fixed heating power per junction and the conductance data show no dip/hump features allow us to conclude directly, and unambiguously, that such peaks represent the transition of the mesa into the normal state. These findings, along with the detailed analyses, go well beyond more preliminary reports. $^{43}$

Beyond this experimental proof, we explored why a backbending $I(V)$, expected in this strong-heating regime, is so robustly and systematically replaced by a narrow peak in $d I / d V$ as $N$ and thus the dissipated power increase. To do so, we needed (a) a realistic heat-transfer model and (b) the full energy and temperature dependence of the superconducting DOS. For (a), previous accounts ${ }^{16,17}$ indicate that Newton's law of cooling [Eq. (2)] is a sufficiently good approximation for uniform heating while numerical integration of the heatflow equation in 1D or quasi-two-dimensional should provide a reasonable approximation for nonuniform temperatures. For (b), the full temperature dependence of the equilibrium tunneling $I(V)$ for intercalated Bi2212 would be best but this has been unattainable with our MCT apparatus. The next best approach comes from STS data on pristine $\mathrm{Bi} 2212$ that was fit of the full temperature range to the $d$-wave DOS [Eq. (1)] with parameters $\Delta(T)$ and $\Gamma(T)$. Using this DOS in the above heat-transfer models always results in backbending for high drive, although nonuniform temperatures provided better qualitative agreement with the trend of our data vs $N$ (Figs. 2 and 6). The remaining discrepancy likely comes from the inadequacy of the $d$-wave DOS (it does not predict the correct low-temperature MCT $I(V)$ in Fig. 6 while an additional momentum-averaging parameter is used in Fig. 4 to get excellent fits to $d I / d V$ for SIN junctions on both pristine and intercalated Bi2212). In particular, the pure $d$-wave $I(V)$ is steeper in the region of instability than the experimental $I(V)$ (see Fig. 6). It is not difficult to imagine that this steeper behavior would be more prone to runaway heating and instability. It may be possible to better simulate the measured $I(V)$ by including directional tunneling (momentum-averaging parameter) but this will be reserved for a future work.

As a practical tool, we introduce a useful metric, the relative width of the coherence peak, $\beta$ that identifies the crossover from near-to-equilibrium to this strong self-heating regime. It is found that other mesa studies with variable self-heating ${ }^{7}$ display the same crossover, i.e., when $\beta>0.3$ the conductance exhibits the strong-coupling dip/hump features but for $\beta<0.15$ the dip/hump is absent.

The extreme difficulty of eliminating heating may imply the need to reinterpret some recent IJJ studies that generally exhibit small or nonexistent DHF in $d I / d V$ and $\beta$ values of $0.03-0.15 .^{8-12}$ Such sharp conductance peaks, often seen in intermediate size mesas, can be easily, but according to our analysis, incorrectly, assigned to $\Delta(T)$. Thus the sharp peaks should not be used to make inferences about $\Delta(T)$ near $T_{\mathrm{c}}$. Further, the narrow peak, as a heating phenomenon, must disappear above $T_{\mathrm{c}}$, and we document that in Fig. 7. Thus this heating effect is easily misinterpreted as a closing of $\Delta(T)$ at $T_{\mathrm{c}}$ and that would necessarily lead (incorrectly) to the conclusion that any pseudogap observed above $T_{\mathrm{c}}$ is extrinsic to superconductivity. We reiterate the incorrectness of conclusions based on such a false interpretation and they are at odds with other spectroscopic measurements. ${ }^{26,27,31}$ While our conclusions place very strict limits on the usefulness of Bi2212 mesas for fundamental studies, it is important for the scientific community to recognize such limitations.

\section{ACKNOWLEDGMENTS}

Work supported by UChicago Argonne, LLC, operator of Argonne National Laboratory, a U.S. Department of Energy, Office of Science Laboratory, operated under Contract No. DE-AC02-06CH11357 and TUBITAK (Scientific and Technical Research Council of Turkey) Project No. 106T053. L.O. acknowledges support from Turkish Academy of Sciences, in the framework of the Young Scientist Award Program (LO/TUBA-GEBIP/2002-1-17). 
*Corresponding author; ckurter@umd.edu

${ }^{1}$ R. Kleiner, F. Steinmeyer, G. Kunkel, and P. Müller, Phys. Rev. Lett. 68, 2394 (1992).

${ }^{2}$ A. Yurgens, Supercond. Sci. Technol. 13, R85 (2000).

${ }^{3}$ A. Yurgens, D. Winkler, T. Claeson, S.-J. Hwang, and J.-H. Choy, Int. J. Mod. Phys. B 13, 3758 (1999).

${ }^{4}$ J. C. Fenton, P. J. Thomas, G. Yang, and C. E. Gough, Appl. Phys. Lett. 80, 2535 (2002); K. Anagawa, Y. Yamada, T. Shibauchi, M. Suzuki, and T. Watanabe, ibid. 83, 2381 (2003).

${ }^{5}$ A. Yurgens, D. Winkler, T. Claeson, S. Ono, and Y. Ando, Phys. Rev. Lett. 90, 147005 (2003); 92, 259702 (2004).

${ }^{6}$ M.-H. Bae, J.-H. Choi, and H.-J. Lee, Appl. Phys. Lett. 86, 232502 (2005).

${ }^{7}$ X. B. Zhu, Y. F. Wei, S. P. Zhao, G. H. Chen, H. F. Yang, A. Z. Jin, and C. Z. Gu, Phys. Rev. B 73, 224501 (2006); S. P. Zhao, X. B. Zhu, and H. Tang, Eur. Phys. J. B 71, 195(2009).

${ }^{8}$ M. Suzuki, T. Watanabe, and A. Matsuda, Phys. Rev. Lett. 82, 5361 (1999); Y. Yamada and M. Suzuki, Phys. Rev. B 66, 132507 (2002).

${ }^{9}$ V. M. Krasnov, M. Sandberg, and I. Zogaj, Phys. Rev. Lett. 94, 077003 (2005).

${ }^{10}$ V. M. Krasnov, A. Yurgens, D. Winkler, P. Delsing, and T. Claeson, Phys. Rev. Lett. 84, 5860 (2000); V. M. Krasnov, ibid. 97, 257003 (2006).

${ }^{11}$ S. O. Katterwe, A. Rydh, and V. M. Krasnov, Phys. Rev. Lett. 101, 087003 (2008).

${ }^{12}$ M. H. Bae, J.-H. Park, J.-H. Choi, H.-J. Lee, and K.-S. Park, Phys. Rev. B 77, 094519 (2008).

${ }^{13}$ K. Anagawa, T. Watanabe, and M. Suzuki, Phys. Rev. B 73, 184512 (2006).

${ }^{14}$ M. Suzuki and T. Watanabe, Phys. Rev. Lett. 85, 4787 (2000).

${ }^{15}$ J. C. Fenton and C. E. Gough, J. Appl. Phys. 94, 4665 (2003); V. M. Krasnov, A. Yurgens, D. Winkler, and P. Delsing, ibid. 89, 5578 (2001).

${ }^{16}$ C. Kurter et al., IEEE Trans. Appl. Supercond. 19, 428 (2009).

${ }^{17}$ V. N. Zavaritsky, Phys. Rev. B 72, 094503 (2005).

${ }^{18}$ L. Ozyuzer, A. E. Koshelev, C. Kurter, N. Gopalsami, Q. Li, M. Tachiki, K. Kadowaki, T. Yamamoto, H. Minami, H. Yamaguchi, T. Tachiki, K. E. Gray, W.-K. Kwok, and U. Welp, Science 318, 1291 (2007).

${ }^{19}$ Q. Huang, J. F. Zasadzinski, K. E. Gray, J. Z. Liu, and H. Claus, Phys. Rev. B 40, 9366 (1989).

${ }^{20}$ N. Miyakawa, J. F. Zasadzinski, L. Ozyuzer, P. Guptasarma, D. G. Hinks, C. Kendziora, and K. E. Gray, Phys. Rev. Lett. 83, 1018 (1999).

${ }^{21}$ L. Ozyuzer, J. F. Zasadzinski, K. E. Gray, C. Kendziora, and N. Miyakawa, Europhys. Lett. 58, 589 (2002).

${ }^{22}$ J. F. Zasadzinski, L. Ozyuzer, L. Coffey, K. E. Gray, D. G. Hinks, and C. Kendziora, Phys. Rev. Lett. 96, 017004 (2006); J. F. Zasadzinski, L. Coffey, P. Romano, and Z. Yusof, Phys. Rev. B 68, 180504 (2003).

${ }^{23}$ J. F. Zasadzinski, L. Ozyuzer, N. Miyakawa, K. E. Gray, D. G.
Hinks, and C. Kendziora, J. Phys. Chem. Solids 63, 2247 (2002).

${ }^{24}$ N. Miyakawa, P. Guptasarma, J. F. Zasadzinski, D. G. Hinks, and K. E. Gray, Phys. Rev. Lett. 80, 157 (1998); L. Ozyuzer, J. F. Zasadzinski, C. Kendziora, and K. E. Gray, Phys. Rev. B 61, 3629 (2000).

${ }^{25}$ J. F. Zasadzinski, L. Ozyuzer, N. Miyakawa, K. E. Gray, D. G Hinks, and C. Kendziora, Phys. Rev. Lett. 87, 067005 (2001).

${ }^{26}$ J. C. Campuzano, H. Ding, M. R. Norman, H. M. Fretwell, M. Randeria, A. Kaminski, J. Mesot, T. Takeuchi, T. Sato, T. Yokoya, T. Takahashi, T. Mochiku, K. Kadowaki, P. Guptasarma, D. G. Hinks, Z. Konstantinovic, Z. Z. Li, and H. Raffy, Phys. Rev. Lett. 83, 3709 (1999).

${ }^{27}$ Ch. Renner, B. Revaz, J.-Y. Genoud, K. Kadowaki, and Ø. Fischer, Phys. Rev. Lett. 80, 149 (1998).

${ }^{28}$ Y. DeWilde, N. Miyakawa, P. Guptasarma, M. Iavarone, L. Ozyuzer, J. F. Zasadzinski, P. Romano, D. G. Hinks, C. Kendziora, G. W. Crabtree, and K. E. Gray, Phys. Rev. Lett. 80, 153 (1998)

${ }^{29}$ S. H. Pan, J. P. O’Neal, R. L. Badzey, C. Chamon, H. Ding, J. R. Engelbrecht, Z. Wang, H. Eisaki, S. Uchida, A. K. Gupta, K.-W. $\mathrm{Ng}$, E. W. Hudson, K. M. Lang, and J. C. Davis, Nature (London) 413, 282 (2001).

${ }^{30}$ R. M. Dipasupil, M. Oda, N. Momono, and M. Ido, J. Phys. Soc. Jpn. 71, 1535 (2002).

${ }^{31}$ A. N. Pasupathy, A. Pushp, K. K. Gomes, C. V. Parker, J. Wen, Z. Xu, G. Gu, S. Ono, Y. Ando, and A. Yazdani, Science 320, 196 (2008).

${ }^{32}$ M. Eschrig and M. R. Norman, Phys. Rev. B 67, 144503 (2003).

${ }^{33}$ V. M. Krasnov, A. E. Kovalev, A. Yurgens, and D. Winkler, Phys. Rev. Lett. 86, 2657 (2001).

${ }^{34}$ J. T. C. Yeh and D. N. Langenberg, Phys. Rev. B 17, 4303 (1978).

${ }^{35}$ H. Won and K. Maki, Phys. Rev. B 49, 1397 (1994).

${ }^{36}$ J. H. Choy, S. J. Hwang, and N. G. Park, J. Am. Chem. Soc. 119, 1624 (1997); K. Ohhashi, T. Horiuchi, S. Nasu, and E. Sugimata, J. Phys. Chem. Solids 65, 603 (2004).

${ }^{37}$ C. Kurter and L. Ozyuzer, J. Optoelectron. Adv. Mater. 7, 415 (2005).

${ }^{38}$ L. Ozyuzer, J. F. Zasadzinski, and K. E. Gray, Cryogenics 38, 911 (1998).

${ }^{39}$ R. Kleiner and P. Muller, Phys. Rev. B 49, 1327 (1994).

${ }^{40}$ K. E. Gray and H. W. Willemsen, J. Low Temp. Phys. 31, 911 (1978).

${ }^{41}$ H. B. Wang, S. Guénon, J. Yuan, A. Iishi, S. Arisawa, T. Hatano, T. Yamashita, D. Koelle, and R. Kleiner, Phys. Rev. Lett. 102, 017006 (2009).

${ }^{42}$ G. S. Nolas, G. A. Slack, D. T. Morelli, T. M. Tritt, and A. C. Ehrlich, J. Appl. Phys. 79, 4002 (1996).

${ }^{43}$ C. Kurter, L. Ozyuzer, J. F. Zasadzinski, D. G. Hinks, and K. E. Gray, IEEE Trans. Appl. Supercond. 17, 2976 (2007). 